\title{
Cavernous hemangioma of the bronchial wall: A systemic-pulmonary vessel fistula
}

Naveen Swami, MCh, Shyam K. S. Thingnam, MCh, and Harkant Singh, MCh, Chandigarh, India

We report a patient presenting with life-threatening hemoptysis and later receiving a diagnosis of systemicpulmonary artery communication, which is an exceptional disease.

\section{CLINICAL SUMMARY}

A 49-year-old man presented to the medical emergency department with complaint of massive hemoptysis without any past history of trauma or surgical intervention. Neither physical examination nor chest radiographic analysis revealed any abnormality. Thereafter, fiberoptic bronchoscopic examination demonstrated a blood clot in the right tracheobronchial tree and one bluish nodular lesion in the right lower lobe bronchus. Subsequently, digital subtraction angiographic analysis revealed that the right bronchial artery is prominent and tortuous, and there was a nidus of $2 \times 2 \mathrm{~cm}$ in the lower lobe region filled through the 2 branches of the bronchial artery and followed by immediate opacification of the descending branch of the right pulmonary artery and then the right inferior pulmonary vein (Figure 1). These findings were suggestive of anomalous communication between the right bronchial artery and the ipsilateral pulmonary artery. Intravascular embolization of the feeding vessel was not carried out because of the associated risk of systemic embolization. Subsequently, right lower lobectomy with interruption of the vascular channel was performed. Intraoperatively, the right lower lobe bronchus lumen revealed blood clots and one mass lesion with bluish discoloration on its wall with extension into the segmental bronchus. Histopathology revealed the submucosal, cavernous, hemangiomatous vascular tissue in the wall of the lower lobe bronchus without any evidence of infective pathology. Postoperative and 1-year follow-up periods remained uneventful.

From the Department of Cardiothoracic and Vascular Surgery, Pgimer, Chandigarh, India.

Received for publication Feb 13, 2008; revisions received March 9, 2008; accepted for publication April 8, 2008.

Address for reprints: Naveen Swami, MCh, CTVS, Pgimer, Chandigarh, India (E-mail: nav_swami_5275@yahoo.com).

J Thorac Cardiovasc Surg 2009;137:1272-3

$0022-5223 / \$ 36.00$

Copyright $@ 2009$ by The American Association for Thoracic Surgery doi:10.1016/j.jtcvs.2008.04.004

\section{DISCUSSION}

Fistulous communication between systemic and pulmonary arteries or pulmonary veins is rare. These abnormalities might be congenital or acquired. The congenital form can be seen with a variety of anomalies, including bronchopulmonary sequestration, hypoplastic lung syndrome, congenital cystic adenomatoid malformation, and absence or proximal interruption of the main pulmonary artery.

A systemic artery might also supply the lung in the absence of these conditions, either in the form of a systemicpulmonary vessel fistula or as a localized blood supply of otherwise normal lung. ${ }^{1}$ In the latter situation, the large systemic artery supplied a portion of one lung and invariably the basal segments of one of the lower lobes either partly or completely.

In 1990, Remy Jardin and Remy ${ }^{2}$ called these malformations "third arterial circulation of the lung", or " nonbronchial systemic arterial circulation.' The anomalous artery is usually solitary and arises from the descending aorta. The most frequent afferent arteries involved in the malformation are the internal thoracic artery and the intercostal arteries.

The etiopathogenesis is unknown; possibly the abnormal development of one or more of the pulmonary ligament arteries might be implicated. Patients can remain asymptomatic or present with recurrent hemoptysis. The age of the presentation ranges from infancy to childhood. Radiologically, these malformations can show either normal or increased vascular markings. ${ }^{1}$ Selective arteriography is the diagnostic procedure of choice because it clearly delineates the anatomy of the lesion, identifying the feeding and draining vessels and the extent of pulmonary parenchymal involvement by the vascular malformation. ${ }^{1}$ Evidence of left-to-right shunting by means of oxygen saturation and positive hydrogen indicator curves can be observed in the pulmonary artery during right heart catheterization, ${ }^{1}$ which we have not performed. In our case fistulous communication was detected by means of digital subtraction angiography, and thus we have not estimated the left-to-right shunt ratio, and the decision for surgical intervention was based on the severity of symptoms. The data on the longterm pathophysiologic effects in the untreated patient is lacking. The uncertainty about the long-term effectiveness of surgical intervention in preventing recurrences supports the conservative approach. Because the risk of surgical intervention is low, the actual and conjectural consideration led us to recommend excisional therapy of the offending malformation. 


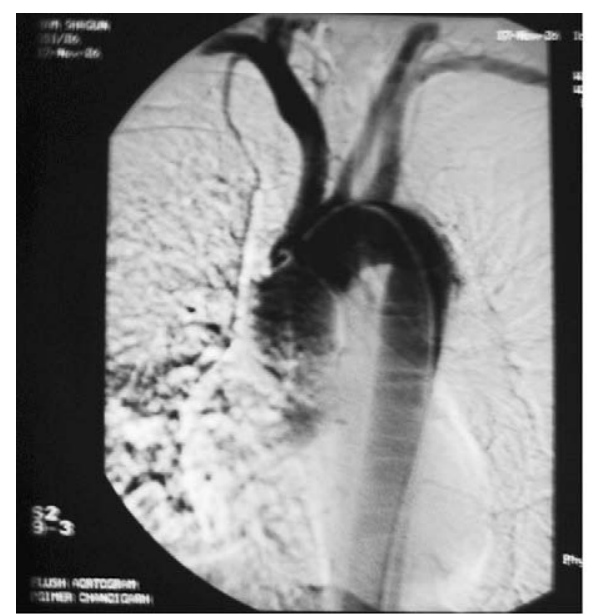

A

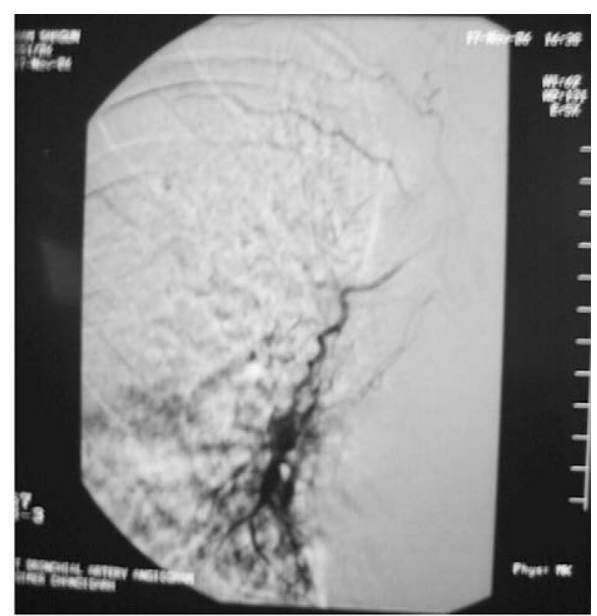

B

FIGURE 1. Digital subtraction angiogram showing filling of a branch of the right bronchial artery from the thoracic aorta (A) and filling of the hemangiomatous portion through the right bronchial artery (B).

\section{References}

1. Brundage BH, Gomez AC, Cheitlin MD, Gmelich JT. Systemic artery to pulmonary vessel fistulas-report of two cases and a review of the literature. Chest. 1972;62:19-23.
2. Riehl G, Chaffanjon P, Frey G, Sessa C, Brichon PY. Postoperative systemic artery to pulmonary vessel fistula: analysis of three cases. Ann Thorac Surg. 2003;76:1873-7.

\title{
Early calcification of a stentless pericardial bioprosthesis in the elderly
}

\author{
Maria Grazia Croccia, MD, ${ }^{\mathrm{a}}$ Stefano Pratali, MD,${ }^{\mathrm{a}}$ Cristina Basso, MD, PhD, ${ }^{\mathrm{b}}$ Giovanni Scioti, MD,${ }^{\mathrm{a}}$ Mila Della Barbera, BSc, PhD, ${ }^{\mathrm{b}}$ \\ Gaetano Thiene, MD, ${ }^{\mathrm{b}}$ and Uberto Bortolotti, MD, ${ }^{\mathrm{a}}$ Pisa and Padova, Italy
}

The Sorin Freedom pericardial valve (SFP, Sorin BiomedicaCardio, Saluggia, Italy), a stentless bioprosthesis made of glutaraldehyde-tanned bovine pericardium, was introduced clinically in $1991 .{ }^{1}$ In 2000 , detoxification treatment with homocysteic acid was added to eliminate glutaraldehyde toxicity and mitigate tissue calcification. The SFP has shown excellent overall results at medium term with no cases of structural failure. ${ }^{2}$ We report the first case of early SFP calcification in an elderly recipient.

\footnotetext{
From the Section of Cardiac Surgery, University of Pisa Medical School, ${ }^{a}$ Pisa, Italy, and Cardiovascular Pathology, University of Padova Medical School, ${ }^{\mathrm{b}}$ Padova, Italy.

Received for publication Feb 28, 2008; accepted for publication March 8, 2008.

Address for reprints: Uberto Bortolotti, MD, Sezione Autonoma di Cardiochirurgia

Universitaria, Dipartimento Cardiotoracico e Vascolare, Via Paradisa 2, 56124

Pisa, Italy (E-mail: u.bortolotti@cardchir.med.unipi.it).

J Thorac Cardiovasc Surg 2009;137:1273-5

$0022-5223 / \$ 36.00$

Copyright (c) 2009 by The American Association for Thoracic Surgery

doi:10.1016/j.jtcvs.2008.03.042
}

\section{CLINICAL SUMMARY}

An 84-year-old woman underwent aortic valve replacement in 2000 with a 25 -mm SFP, implanted with 3 continuous sutures of 4-0 polypropylene to secure the inflow and outflow rims; intraoperative echocardiography showed a competent valve. Approximately 6 years later she was hospitalized for acute pulmonary edema. A harsh precordial systolic murmur was audible, and electrocardiography showed sinus rhythm and left ventricular hypertrophy. Echocardiography revealed calcified valve cusps with a peak transvalvular gradient of $100 \mathrm{~mm} \mathrm{Hg}$ and a valve area of $0.8 \mathrm{~cm}^{2}$. No evident risk factors for bioprosthetic calcification were detected; renal function was normal (serum creatinine $0.78 \mathrm{mg} / \mathrm{dL}$ ) with no hyperlipidemia, and hyperparathyroidism was excluded by normal calcium and phosphate values. At reoperation, the prosthesis appeared incorporated into the aortic wall; all cusps were calcified (Figure 1), and calcification extended to the outflow of the prosthesis preventing its complete removal. The 3 cusps were separately excised leaving a rigid diminutive aortic 